\title{
An Approximation method for Power Indices for Voting Games
}

\author{
Shaheen S. Fatima ${ }^{1} \quad$ Michael Wooldridge $^{2} \quad$ Nicholas R. Jennings $^{3}$ \\ ${ }^{1}$ Department of Computer Science \\ Loughborough University, Loughborough, LE11 3TU, UK \\ s.s.fatimallboro.ac.uk \\ ${ }^{2}$ Department of Computer Science \\ University of Liverpool, Liverpool, L69 3BX, UK \\ mjw@csc.liv.ac.uk \\ ${ }^{3}$ School of Electronics and Computer Science \\ University of Southampton, Southampton SO17 1BJ, UK \\ nrjeecs.soton.ac.uk
}

\begin{abstract}
The Shapley value and Banzhaf index are two well known indices for measuring the power a player has in a voting game. However, the problem of computing these indices is computationally hard. To overcome this problem, we analyze approximation methods for computing these indices. Although these methods have polynomial time complexity, finding an approximate Shapley value using them is easier than finding an approximate Banzhaf index. We also find the absolute error for the methods and show that this error for the Shapley value is lower than that for the Banzhaf index.
\end{abstract}

\section{Introduction}

Coalition formation is a key form of interaction in multi-agent systems. It is the process of joining together two or more agents so as to achieve goals that individuals on their own cannot, or to achieve them more efficiently [10]. Often, in such situations, there is more than one possible coalition, and the agents/ players must decide how to form a coalition and how to split the gains of cooperation between the members of a coalition. In this context, cooperative game theory offers a number of solution concepts such as core, kernel, and stable solution [10]. A number of multiagent systems researchers have used and extended these solutions to facilitate automated coalition formation [16, $17,14,13]$. A key problem, in the context of multi-agent systems, is to study the computational aspects of the solutions that game theory provides. For example, [4] shows that the problem of finding the core is NP-complete. Another problem with these solutions is that, often there is more than one possible solution.

In order to overcome the problem of multiple solutions, Shapley proposed a solution called the Shapley value [15]. The Shapley value not only provides a unique solution to coalitional games but also provides a measure of how much influence or power a player has in determining the outcome of a game. The higher a player's Shapley value, the more control he has in determining the outcome of a game. Thus the Shapley value 
can be viewed as an index for measuring the power of players in a game. Like Shapley value, the Banzhaf index [2] is another way of measuring a player's power. However, a key drawback of both these power indices is that computing them for voting games ${ }^{1}$ is, in general, \#P-complete $[5,12]$. In other words, it is practically infeasible to try to compute the exact Shapley value or Banzhaf index. Hence, in order to overcome this computational complexity we present a new randomized method for finding an approximation for these indices.

The time complexity of the proposed approximation methods is polynomial in the number of players. Now, the quality of an approximation is evaluated in terms of its error of approximation. To this end, we find the absolute error for the proposed methods and show that this error for the Shapley value is lower than that for the Banzhaf index.

Although some approximation methods for the Shapley value have been proposed in the past, to our knowledge, there has been no study of their performance in terms of the approximation errors (see Section 5 for details). This paper not only provides new approximation methods, but also analyzes them in terms of their errors.

The rest of the paper is organised as follows. Section 2 provides the background to voting games and power indices. In Section 3 we present our approximation methods. In Section 4 we analyze their absolute error. Section 5 discusses related literature. Section 6 concludes.

\section{Background}

A coalitional game $\langle N, v\rangle$, consists of:

1. a finite set, $N=\{1,2, \ldots, n\}$ of players, and

2. a function, $v$, that associates with every non-empty subset $S$ of $N$ (i.e., a coalition) a real number $v(S)$ the worth of $S$ that corresponds to it.

For each coalition $S, v(S)$ is the total payoff that is available for division among the members of $S$.

\subsection{Weighted voting game}

A weighted voting game $G=\langle N, v\rangle$ is a game such that [10]:

$$
v(S)=\left\{\begin{array}{l}
1 \text { if } w(S) \geq q \\
0 \text { otherwise }
\end{array}\right.
$$

for some $q \in \mathbb{R}_{+}$and $w_{i} \in \mathbb{R}_{+}$, where:

$$
w(S)=\sum_{i \in S} w_{i}
$$

for any coalition $S$. Thus $w_{i}$ is the number of votes that player $i$ has and $q$ is the number of votes needed to win the game (i.e., the quota). This game is denoted as $\left\langle q ; w_{1}, \ldots, w_{n}\right\rangle$.

\footnotetext{
${ }^{1}$ Voting games are an important mechanism for agents to reach consensus.
} 


\subsection{Weighted $k$-majority game}

For the set of $n$ players, a weighted $k$-majority game $\left(v_{1} \wedge \ldots \wedge v_{k}\right)$ is a game where $v_{t}=\left[q^{t} ; w_{1}^{t}, \ldots, w_{n}^{t}\right], 1 \leq t \leq k$ are weighted voting games and

$$
\left(v_{1} \wedge \ldots \wedge v_{k}\right)(S)=\left\{\begin{array}{l}
1 \text { if } w^{t}(S) \geq q^{t} \text { for } 1 \leq t \leq k \\
0 \text { otherwise }
\end{array}\right.
$$

where $w^{t}(S)=\sum_{i \in S} w_{i}^{t}$.

\subsection{Power indices}

A power index for a voting game is a way of measuring a player's voting power. A player's power is his ability to turn a losing coalition into a winning one. The Shapley value and Banzhaf index are examples of power indices. The Banzhaf index, in turn, has two versions: the absolute Banzhaf index and the normalised Banzhaf index. For a voting game $G=\langle N, v\rangle$, these indices are defined as follows [15,2].

The marginal contribution of player $i$ to coalition $S$ with $i \notin S$ is a function $\Delta_{i} v$ that is defined as follows:

$$
\Delta_{i} v(S)=v(S \cup\{i\})-v(S)
$$

This means a player's marginal contribution to a coalition $S$ is the increase in the value of $S$ as a result of $i$ joining it. A player that makes a higher marginal contribution, on average, has a higher Shapley value. Specifically, a player's Shapley value is defined in terms of its marginal contribution as follows [15]:

Definition 1. The Shapley value $\left(\varphi_{i}\right)$ of the game $\langle N, v\rangle$ for player $i$ is the average of its marginal contribution to all possible coalitions:

$$
\phi_{i}=\sum_{S \subset N} \frac{|S| !(n-|S|-1) !}{n !} \times \Delta_{i} v(S)
$$

Note that for a voting game $\left(\left\langle q ; w_{1}, \ldots, w_{n}\right\rangle\right)$, a player's marginal contribution is either zero or one. This is because the value of any coalition is either zero or one. A coalition with value zero is called a "losing coalition" and with value one a "winning coalition". If a player's entry to a coalition changes it from losing to winning, then the player's marginal contribution for that coalition is one; otherwise it is zero. A coalition $S$ is said to be a swing for player $i$ if $S$ is losing but $S \cup\{i\}$ is winning.

For player $i \in N$, let $\eta_{i}$ denote the number of swings, i.e.,:

$$
\eta_{i}=\sum_{T_{i}} 1
$$

where $T_{i}$ is a losing coalition but $T_{i} \cup\{i\}$ is winning. The two versions of Banzhaf index are defined by expressing $\eta_{i}$ over different denominators.

Definition 2. For player $i$, the absolute Banzhaf index $\left(\beta_{i}\right)$ is defined as [2]:

$$
\beta_{i}=\eta_{i} / 2^{n-1}
$$


Definition 3. For player $i$, the normalized Banzhaf index $\left(\lambda_{i}\right)$ is defined as [2]:

$$
\lambda_{i}=\eta_{i} / \Sigma_{i=1}^{n} \eta_{i}
$$

Note that the normalized Banzhaf index sums to unity over the players: $\sum \lambda_{i}=1$.

The problem of computing the Shapley value or the Banzhaf index for voting games is \#P-complete $[5,12]$. In order to overcome this problem, we present new approximation methods to find these indices.

\section{Approximate Power Indices}

The methods we propose are an extension of the one presented in [7]. In more detail, [7] is an approximation for the Shapley value for weighted voting games. Here we extend this to find approximates for the Shapley value and the Banzhaf index for both wieghted voting games and $k$-majority games. Section 3.1 deals with methods for weighted voting games and Section 3.2 with those for $k$-majority games.

\subsection{Weighted Voting Game}

The intuition behind the method proposed in [7] is as follows. As per Definition 1, in order to find a player's Shapley value, we first need to find his marginal contribution to all possible coalitions. For $n$ players, there are $2^{n-1}$ possible coalitions. Finding a player's marginal contribution to each of these $2^{n-1}$ possible coalitions is computationally infeasible. So instead of finding the marginal contribution to each possible coalition, this method finds a player's expected marginal contribution to random coalitions of size $X$ where $1 \leq X \leq n$. This is done by using the approximation rule $\mathcal{R}_{1}$ which is defined as follows.

Let the players' weights in $N$ be defined by any probability distribution function. Irrespective of the actual form of this function, let $\mu$ be the mean weight for the set of players and $\nu$ be the variance in the players' weights. From this set $(N)$ if we randomly draw a sample, then the approximate sum of the players' weights in the sample is given by the following rule [8]:

$\mathcal{R}_{1}$ : If $w_{1}, w_{2}, \ldots, w_{X}$ is a random sample of size $X$ drawn from any distribution with mean $\mu$ and variance $\nu$, then the sample sum has an approximate Normal distribution, $\mathcal{N}$, with mean $X \mu$ and variance $\frac{\nu}{X}$ (the larger the $n$ the better the approximation ${ }^{2}$ ).

We know, from Definition 1, that the Shapley value for a player is the expectation $(E)$ of its marginal contribution to a coalition that is chosen randomly. The above rule is used to determine the Shapley value as follows.

For player $i$ with weight $w_{i}$, let $\bar{\varphi}_{i}$ denote the approximate Shapley value. Also, let $X$ denote the size of a random sample drawn from $N$. The marginal contribution of player $i$ to this random sample is one if the total weight of the $X$ players in the

\footnotetext{
${ }^{2}$ Also, for large $X$, any measurement done on a sample drawn with replacement is the same as that for a sample drawn without replacement [8].
} 


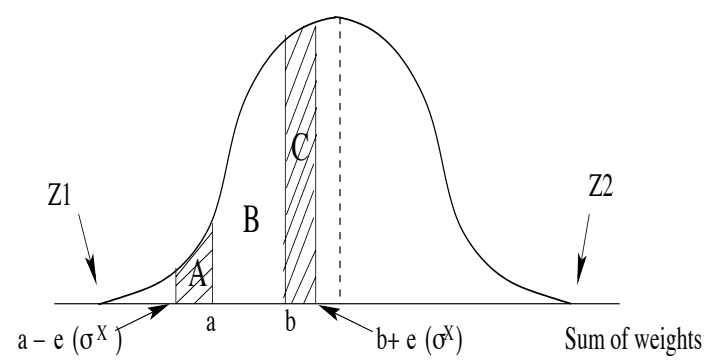

Fig. 1. A normal distribution for the sum of players' weights in a coalition of size $X$.

sample is greater than or equal to $a=q-w_{i}$ but less than $b=q-\epsilon$ (where $\epsilon$ is an inifinitesimally small quantity). Otherwise, its marginal contribution is zero. Thus, the expected (approximate) marginal contribution of player $i$ (denoted $E \Delta_{i}^{X}$ ) to the sample coalition is the area under the curve defined by $\mathcal{N}\left(X \mu, \frac{\nu}{X}\right)$ in the interval $[a, b]$. This area is shown as the region $B$ in Figure 1 (the dotted line in the figure is $X \mu$ ). Hence $i$ 's approximate marginal contribution to $X$ is:

$$
E \Delta_{i}^{X}=\frac{1}{\sqrt{(} 2 \pi \nu / X)} \int_{a}^{b} e^{-X \frac{(x-X \mu)^{2}}{2 \nu}} d x .
$$

And, as per Definition 1, $i$ 's approximate Shapley value (denoted $\bar{\varphi}_{i}$ ) is the average of his expected marginal contribution to all possible coalitions:

$$
\bar{\varphi}_{i}=\frac{1}{n} \sum_{X=1}^{n} E \Delta_{i}^{X}
$$

The time complexity of this method is $\mathcal{O}(n)$ [7].

We now extend this method to find the Banzhaf index. For a game of $n$ players, let $T$ denote the number of possible coalitions of $X$ players, i.e., $T=C(n, X)$ is the number of combinations of $X$ items drawn from a set of $n$ items. Given this, player $i$ 's total approximate marginal contribution to all coalitions of size $X$ is $C(n, X) \times E \Delta_{i}^{X}$ where $E \Delta_{i}^{X}$ is as computed in Equation 6. In other words, $i$ 's approximate number of swings for coalitions of size $X$ is:

$$
\bar{\eta}_{i}^{X}=C(n, X) \times E \Delta_{i}^{X}
$$

Hence, $i$ 's approximate number of swings to coalitions of all possible sizes $(1 \leq X \leq$ $n$ ) is:

$$
\bar{\eta}_{i}=\sum_{X=1}^{n} \bar{\eta}_{i}^{X}
$$

As per Equation 4, $i$ 's approximate absolute Banzhaf index $\left(\bar{\beta}_{i}\right)$ is:

$$
\bar{\beta}_{i}=\bar{\eta}_{i} / 2^{n-1}
$$


And as per Equation 5, $i$ 's approximate normalised Banzhaf index $\left(\bar{\lambda}_{i}\right)$ is:

$$
\bar{\lambda}_{i}=\bar{\eta}_{i} / \delta
$$

where $\delta=\sum_{i=1}^{n} \bar{\eta}_{i}$.

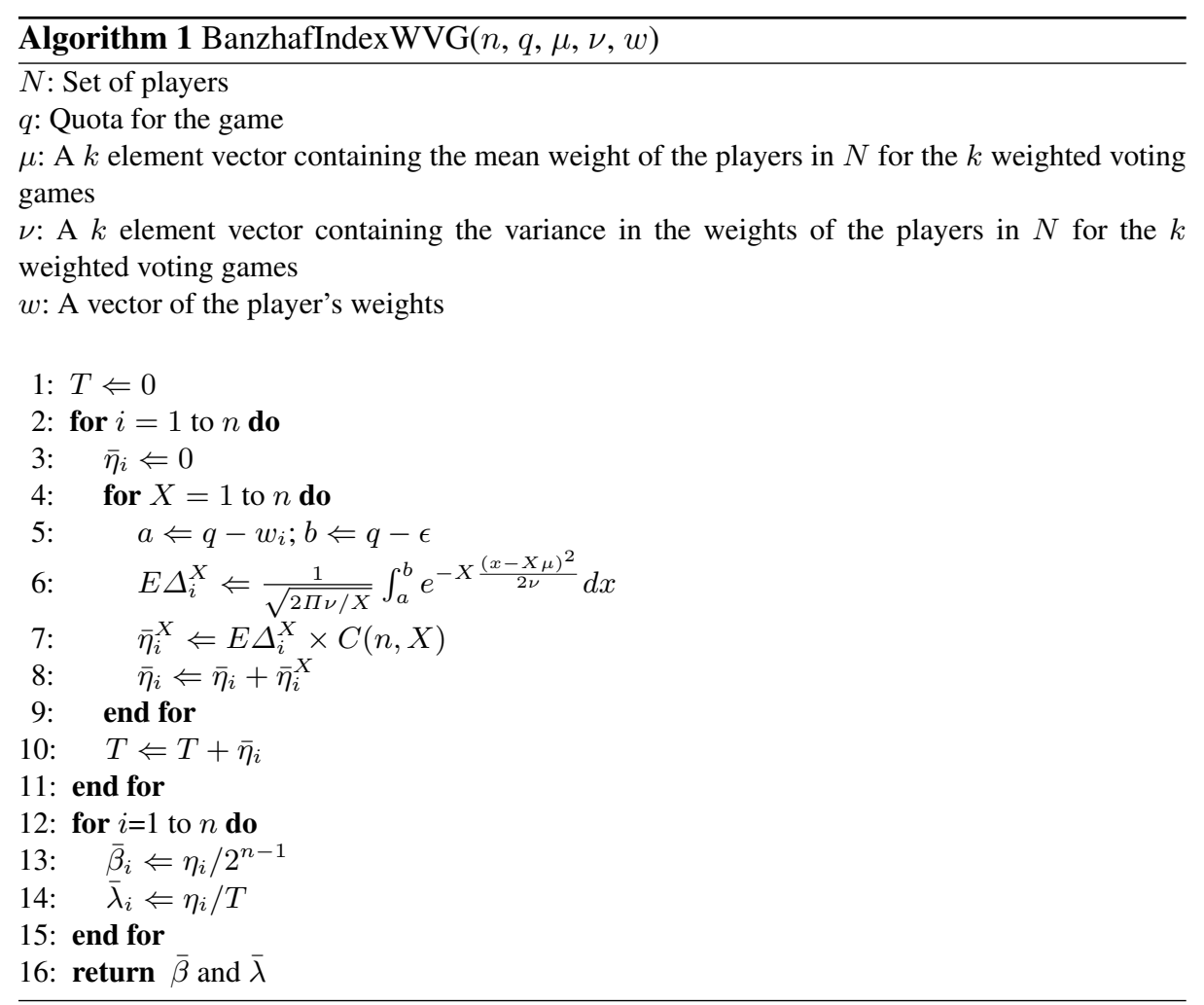

The above steps are described in Algorithm 1. In more detail, Step 1 does the initialization. In Step 2, we vary $X$ between 1 and $n$ and repeatedly do the following. Step 3 is another initialization. In Step 4, we repeatedly do the followig. We find player $i$ 's approximate marginal contribution to the random coalition of size $X$. In Step 7, we use Equation 6 to find the approximate number of player $i$ 's swings for coalitions of size $X$. In Step 8, we do the same for coalitions of all possible sizes. In Step 10, we find the approximate sum of the swings for all the $n$ players. Finally, in Step 13 (14), we find $i$ 's approximate absolute (normalized) Banzhaf index.

Theorem 1. The time to compute $\bar{\beta}_{i}$ is $\mathcal{O}\left(n^{2}\right)$, and that for $\bar{\lambda}_{i}$ is $\mathcal{O}\left(n^{3}\right)$.

Proof. Since the time to compute $X !$ is $\mathcal{O}(X)$ and the time to compute $E \Delta_{i}^{X}$ (as per Equation 6) is $\mathcal{O}(1)$, the time to compute $\bar{\eta}_{i}^{X}$ is $\mathcal{O}(n)$ (see Equation 8). From Equation 9 we get the time to compute $\bar{\eta}_{i}$ as $\mathcal{O}\left(n^{2}\right)$, and from Equation 10 we get the time 
to compute $\bar{\beta}_{i}$ as $\mathcal{O}\left(n^{2}\right)$. Note that $E \Delta_{i}^{X}$ depends on the weight of player $i$, so it is different for different players. However, $C(n, X)$ and $X !$ are the same for all the players. Thus, we need to find $C(n, X)$ and $X$ ! just once and reuse these values to compute the Banzhaf index for all the players. So once we find $\bar{\beta}_{i}$ for a player $i$, the time to find $\bar{\eta}_{j}^{X}$ for $j \neq i$ is $\mathcal{O}(1)$. So the time to find $\bar{\eta}_{j}$ is $\mathcal{O}(n)$. Given this, the time to find $\bar{\beta}_{j}$ for all players such that $i \neq j$ is $\mathcal{O}\left(n^{2}\right)$. It follows that $\delta=\sum \bar{\eta}_{i}$ can be found in time $\mathcal{O}\left(n^{3}\right)$ and so can $\bar{\lambda}_{i}$.

\section{2 $k$-Majority Voting Game}

We now extend the method described in [7] to $k$-majority games. The intuition behind the proposed method is as follows. As described in Section 2.2, a $k$-majority game is defined in terms of $k$ weighted voting games $v_{j}(1 \leq j \leq k)$. Given this definition, we first find a player's approximate marginal contribution to $v_{j}(1 \leq j \leq k)$ using the method in [7]. Then on the basis of these $k$ marginal contributions, we find an approximate marginal contribution for a $k$-majority game as follows.

For a random coalition $S_{X}$ of size $X$, the approximate marginal contribution of player $i$ to the game $v_{1} \wedge \ldots \wedge v_{k}$ is 1 if the following conditions hold:

1. there is at least one game $v_{j}(1 \leq j \leq k)$ for which $i$ is the swing player, and

2. for each game $v_{j}$, the value of $S_{X} \cup\{i\}$ is 1 .

We first introduce some notation to formalise the above conditions and then find an approximate Shapley value. Let $S_{X}$ be a random sample (of size $X$ ) drawn from $N$. For game $v_{t}$ and player $i$, let $P L_{i}^{t}\left(S_{X}\right)$ (where $S_{X} \subset N-\{i\}$ ) denote the probability that the coalition $S_{X}$ is losing but $S_{X} \cup\{i\}$ is winning (i.e., for game $v_{t}$, the probability that the expected marginal contribution of $i$ to $S_{X}$ is 1 ). Also, for game $v_{t}$, let $P W^{t}\left(S_{X}\right)$ denote the probability that the coalition $S_{X}$ is winning (i.e., the probability that marginal contribution of $i$ to $S_{X}$ is 0 ). Finally, for game $v_{t}$, let $\mu^{t}$ denote the mean weight of the players, $\nu^{t}$ the variance in their weights, and $q^{t}$ the quota. Then, for a $k$-majority game, $i$ 's expected marginal contribution to $S_{X}$ is:

$$
k E \Delta_{i}^{X}=\sum_{j=1}^{k}\left(\prod_{f=1}^{j} P L_{i}^{f}\left(S_{X}\right) \times \prod_{g=j+1}^{k} P W^{g}\left(S_{X}\right)\right)
$$

where $P L_{i}^{t}\left(S_{X}\right)$ is the area under the normal distribution $N\left(\mu^{t}, \nu^{t}\right)$ between the limits $q^{t}-w_{i}^{t}$ and $q^{t}-\epsilon$ :

$$
P L_{i}^{t}\left(S_{X}\right)=\frac{1}{\sqrt{\left(2 \pi \nu^{t} / X\right)}} \int_{q^{t}-w_{i}^{t}}^{q^{t}-\epsilon} e^{-X \frac{\left(x-X \mu^{t}\right)^{2}}{2 \nu^{t}}} d x
$$

and $P W^{t}\left(S_{X}\right)$ is the area under the normal distribution $N\left(\mu^{t}, \nu^{t}\right)$ between the limits $q^{t}$ and $\infty$ :

$$
P W^{t}\left(S_{X}\right)=\frac{1}{\sqrt{\left(2 \pi \nu^{t} / X\right)}}\left(\int_{0}^{q^{t}-w_{i}^{w}-1} e^{-X \frac{\left(x-X \mu^{t}\right)^{2}}{2 \nu^{t}}} d x+\int_{q^{t}}^{\infty} e^{-X \frac{\left(x-X \mu^{t}\right)^{2}}{2 \nu^{t}}} d x() 4\right)
$$


Given $P L_{i}^{t}\left(S_{X}\right)$ and $P W^{t}\left(S_{X}\right)$, the approximate Shapley value (as per Definition 1) for player $i$ for a $k$-majority game is:

$$
\bar{\varphi}_{i}^{k}=\frac{1}{n} \sum_{X=1}^{n} k E \Delta_{i}^{X}
$$

The above steps are described in Algorithm 2. We now present the time complexity of this method.

Theorem 2. The time complexity of Algorithm 2 is $\mathcal{O}\left(k^{2} n\right)$.

Proof. The time to execute the for loop in Step 4 of Algorithm 2 is $\mathcal{O}\left(k^{2}\right)$. Since this for loop is within the for loop of Step 2 (which is executed $n$ times), the time complexity of Algorithm 2 is $\mathcal{O}\left(k^{2} n\right)$.

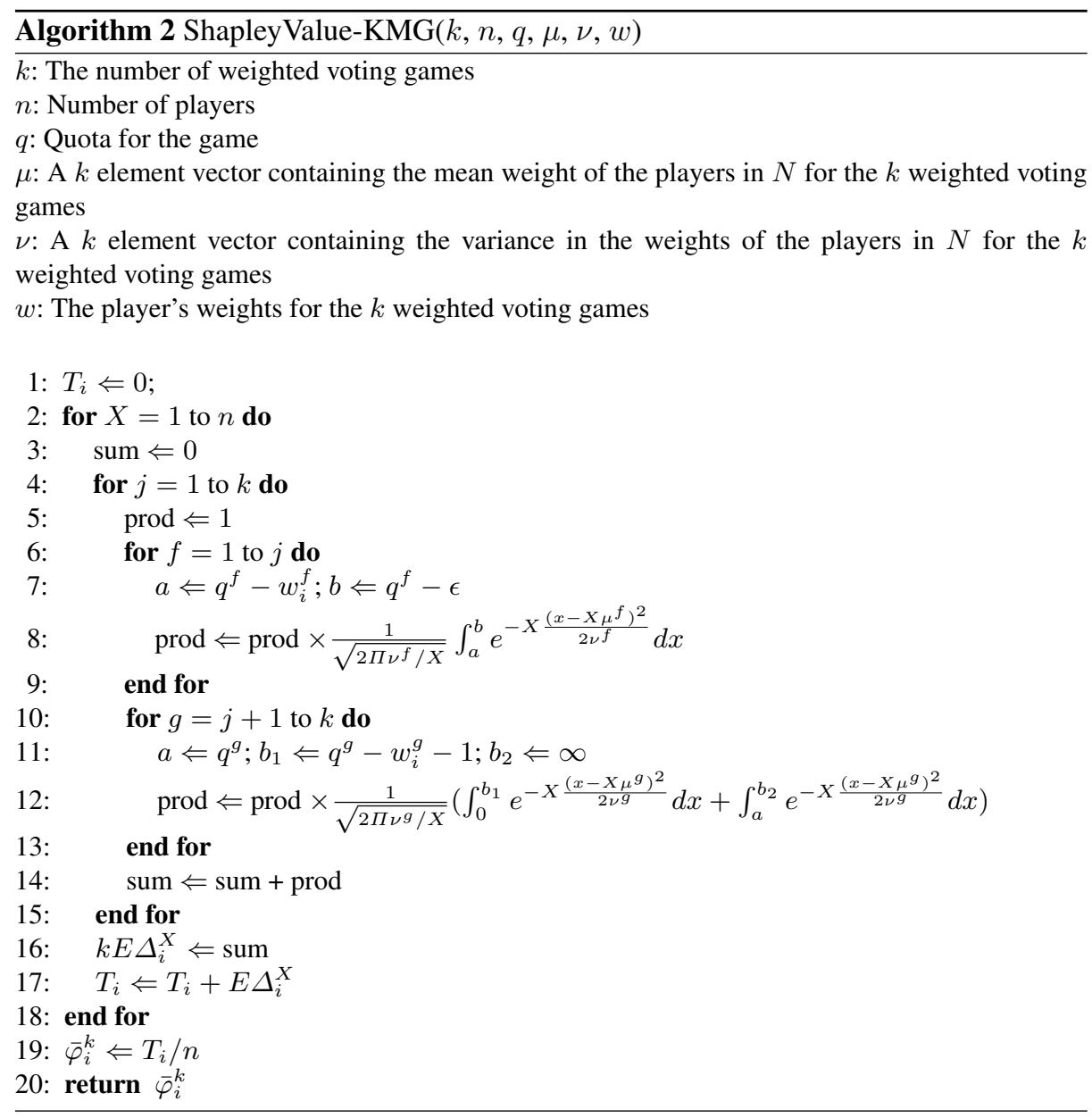


We now extend the method described in Algorithm 1 (for Banzhaf index) to $k$-majority games. For player $i$, let $k \bar{\eta}_{i}^{X}$ denote the approximate number of swings for coalitions of size $X$. Then from Equation 8, we have the following:

$$
k \bar{\eta}_{i}^{X}=C(n, X) \times k E \Delta_{i}^{X}
$$

where $k E \Delta_{i}^{X}$ is as computed in Equation 12. Substituting Equation 12 in Equation 16 we get $k \bar{\eta}_{i}^{X}$. For player $i$, let $k \bar{\eta}_{i}$ be the approximate number of swings for coalitions of all possible sizes. Also, for player $i$, let $\bar{\beta}_{i}^{k}$ and $\bar{\lambda}_{i}^{k}$ denote the approximate absolute and normalised Banzhaf indices respectively. Then, player $i$ 's approximate number of swings over coalitions of all possible sizes is:

$$
k \bar{\eta}_{i}=\sum_{X=1}^{n} k \bar{\eta}_{i}^{X}
$$

As per Equation 4, $i$ 's approximate absolute Banzhaf index $\left(\bar{\beta}_{i}^{k}\right)$ is:

$$
\bar{\beta}_{i}^{k}=k \bar{\eta}_{i} / 2^{n-1}
$$

And as per Equation 5, $i$ 's approximate normalised Banzhaf index $\left(\bar{\lambda}_{i}^{k}\right)$ is:

$$
\bar{\lambda}_{i}^{k}=k \bar{\eta}_{i} / \delta^{k}
$$

where $\delta^{k}=\sum_{i=1}^{n} k \bar{\eta}_{i}$.

The above steps are detailed in Algorithm 3.

Theorem 3. The time to compute $\bar{\beta}_{i}^{k}$ is $\mathcal{O}\left(k^{2} n^{2}\right)$ and that for $\bar{\lambda}_{i}^{k}$ is $\mathcal{O}\left(n^{3} k^{2}\right)$.

Proof. As per Equation 12, the time to find $k E \Delta_{i}^{X}$ is $\mathcal{O}\left(k^{2}\right)$. Also, as per Equation 16, the time to find $k \bar{\eta}_{i}^{X}$ is $\mathcal{O}\left(n k^{2}\right)$. From Equation 17, we get the time to find $k \bar{\eta}_{i}$ as $\mathcal{O}\left(n^{2} k^{2}\right)$. From Equation 18, we know that the time find $\bar{\beta}_{i}^{k}$ the same as the time to find $k \bar{\eta}_{i}$. Given this, the time to compute $\delta^{k}$ is n times the time to compute $k \bar{\eta}_{i}$. Hence, from Equation 19, we get the time to compute $\bar{\lambda}_{i}^{k}$ as $\mathcal{O}\left(n^{3} k^{2}\right)$.

Now, the quality of an approximation method is evaluated on the basis of its running time and also its approximation error. To this end, the following section conducts error analysis for the proposed methods. 


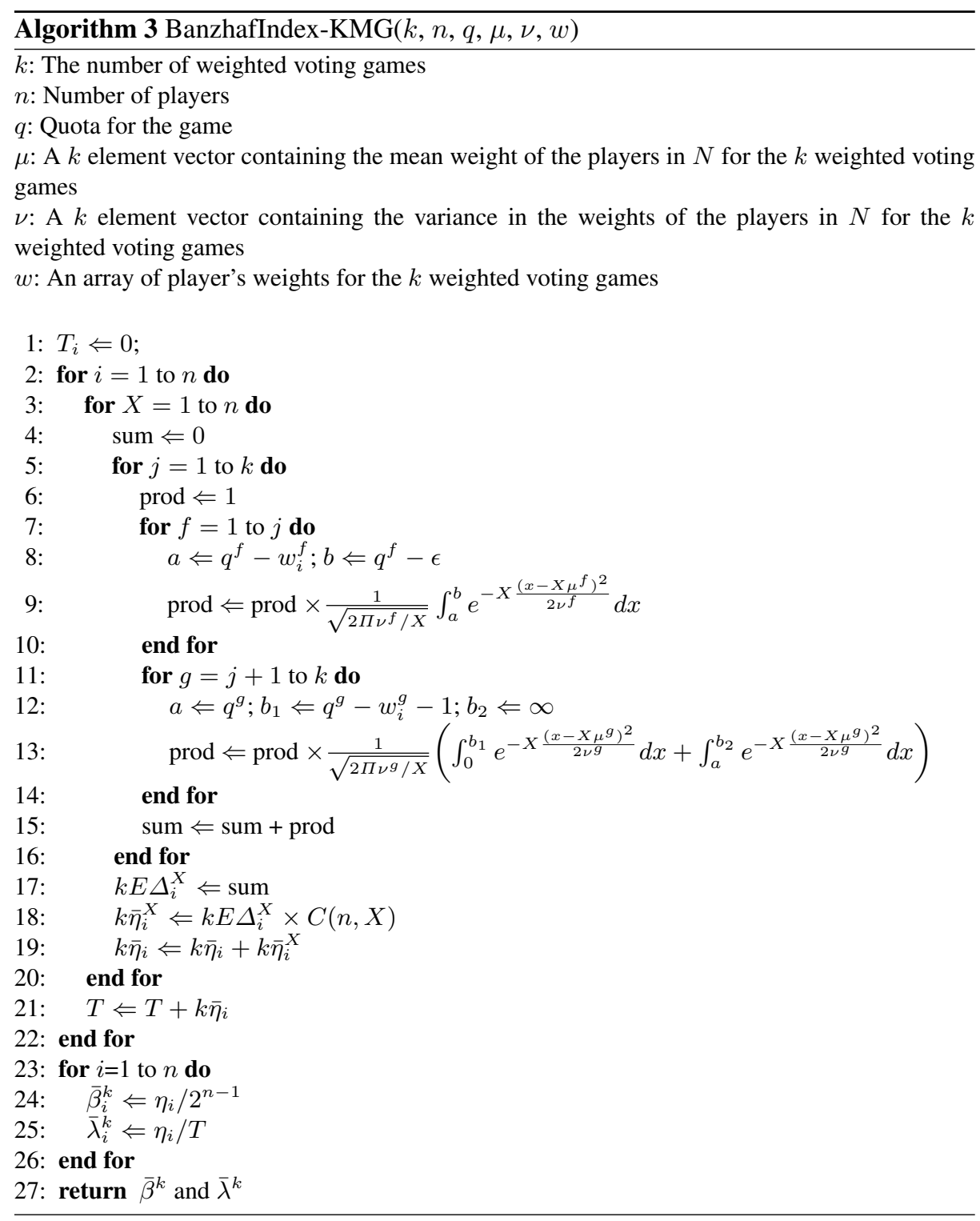

\section{Error Analysis}

We first formalize the idea of error and then derive the formula for measuring the error in the approximate power indices of Section 3. The concept of error relates to a measurement made of a quantity which has an exact value $[18,3]$. Obviously, it cannot be determined exactly how far off a measurement is from the exact value; if this could be done, it would be possible to just give the more accurate, corrected value. Thus, error 
has to do with uncertainty in measurements that nothing can be done about. However, although it is not possible to do anything about such an error, it can be characterized in terms of two essential components $[18,3]$ :

1. a numerical value giving the best "estimate" possible of the quantity measured, and

2. the degree of uncertainty associated with this estimated value.

For example, if the estimate of a quantity is $x$ and the uncertainty is $e(x)$ the quantity would lie in $x \pm e(x)$. For sampling based methods, uncertainty is characterized in terms of standard error [18] which is analogous to the algorithmic term absolute error. This error is equal to the absolute difference between the approximate and its exact counterpart [1]. We first find this error for our approximate Shapley value and then compare it with the error for our approximate Banzhaf index.

\subsection{Absolute error}

Standard error, which we use to measure the absolute error, is defined as follows [18, 3]:

Definition 4. Standard error is defined as the standard deviation for a set of measurements divided by the square root of the number of measurements.

Given this definition, for a weighted voting game, let $e\left(\sigma^{X}\right)$ be the absolute error in the approximate sum of weights for a random coalition of size $X$ where:

$$
\begin{aligned}
e\left(\sigma^{X}\right) & =\sqrt{(} \nu / X) / \sqrt{(} X) \\
& =\sqrt{(}(\nu) / X .
\end{aligned}
$$

Then let $e\left(E \Delta_{i}^{X}\right)$ denote the error in the approximate marginal contribution for player $i$ (given in Equation 6). This error is obtained by propagating the error in Equation 20 to the error in a player's expected marginal contribution given in Equation 6. In Equation 6, $a$ and $b$ are the lower and upper limits for the sum of the players' weights for a coalition of size $X$. Since the error in this sum is $e\left(\sigma^{X}\right)$, the actual values of $a$ and $b$ lie in the intervals $a \pm e\left(\sigma^{X}\right)$ and $b \pm e\left(\sigma^{X}\right)$ respectively. Hence, the error in Equation 6 is either the probability that the sum lies between the limits $a-e\left(\sigma^{X}\right)$ and $a$ (i.e., the area under the curve defined by $\mathcal{N}\left(X \mu, \frac{\nu}{X}\right)$ between $a-e\left(\sigma^{X}\right)$ and $a$, which is the shaded region $A$ in Figure 1) or the probability that the sum of weights lies between the limits $b$ and $b+e\left(\sigma^{X}\right)$ (i.e., the area under the curve defined by $\mathcal{N}\left(X \mu, \frac{\nu}{X}\right)$ between $b$ and $b+e\left(\sigma^{X}\right)$, which is the shaded region $C$ in Figure 1). More specifically, the error is at most the maximum of these two probabilities:

$$
\begin{array}{r}
e\left(E \Delta_{i}^{X}\right)=\frac{1}{\sqrt{(2 \pi \nu / X)} \times \operatorname{MAX}}\left(\int_{a-e\left(\sigma^{X}\right)}^{a} e^{-X \frac{(x-X \mu)^{2}}{2 \nu}} d x,\right. \\
\left.\int_{b}^{b+e\left(\sigma^{X}\right)} e^{-X \frac{(x-X \mu)^{2}}{2 \nu}} d x\right)
\end{array}
$$

On the basis of the above error, we find the error in the Shapley value by using the following standard error propagation rules. Let $x$ and $y$ be two random variables with 
errors $e(x)$ and $e(y)$ respectively. Then, from [18] we have the following propagation rules:

$\mathrm{R} 1$ The error in the random variable $z=x+y$ is:

$$
e(z)=e(x)+e(y)
$$

R2 If $z=k x$ where the constant $k$ has no error, then the error in $z$ is:

$$
e(z)=|k| e(x)
$$

$\mathrm{R} 3$ The error in the random variable $z=x \times y$ is:

$$
e(z)=e(x)+e(y)
$$

\subsection{Absolute error for weighted voting games}

Using the above rules, the error in the Shapley value (given in Equation 7) is obtained by propagating the error in Equation 21 to all coalitions between the sizes $X=1$ and $X=n$. This error (denoted $\left.e\left(\bar{\varphi}_{i}\right)\right)$ is:

$$
e\left(\bar{\varphi}_{i}\right)=\frac{1}{n} \sum_{X=1}^{n} e\left(E \Delta_{i}^{X}\right)
$$

Note that we are finding the absolute error for the Shapley value. Here, it is interesting to note that a related concept for characterising the quality of approximation is performance ratio. Roughly speaking, this is the ratio of an approximate solution and its exact counterpart [1]. The problem of approximating the Shapley value such that the approximation ratio is bounded by a constant is intractable unless $\mathrm{P}=\mathrm{NP}$ [6]. In future, it would be interesting to obtain a similar result for the absolute error as well.

We now turn to the error in the approximate Banzhaf index. Using the error propagation rules (R1, R2, and R3), we get the error in the $\bar{\eta}_{i}^{X}$ (see Equation 8) as:

$$
e\left(\bar{\eta}_{i}^{X}\right)=e\left(E \Delta_{i}^{X}\right) \times C(n, X) .
$$

Given Equation 23, the error in $\bar{\eta}_{i}$ (see Equation 9) is:

$$
e\left(\bar{\eta}_{i}\right)=\sum_{X=1}^{n} e\left(\bar{\eta}_{i}^{X}\right)
$$

From Equations 24 and 10, we get the error in $\bar{\beta}_{i}$ as:

$$
e\left(\bar{\beta}_{i}\right)=e\left(\bar{\eta}_{i}\right) / 2^{n-1}
$$

And, as per Equaton 11, the error in $\bar{\lambda}_{i}$ is:

$$
e\left(\bar{\lambda}_{i}\right)=e\left(\bar{\eta}_{i}\right)+\sum_{i=1}^{n} e\left(\bar{\eta}_{i}\right)
$$

The above equations lead to the following observation for our methods:

Observation. For a given weighted voting game, we have the following relationship: the approximation error in a player's normalized Banzhaf index is higher than the error in its absolute Banzhaf index and the error in its Shapley value $\left(e\left(\bar{\lambda}_{i}\right)>e\left(\bar{\varphi}_{i}\right)\right.$ and $\left.e\left(\bar{\lambda}_{i}\right)>e\left(\bar{\beta}_{i}\right)\right)$. 


\subsection{Error for $k$-majority games}

On the basis of the results of Section 4.2, we now analyze the error for $k$-majority games. Before doing so, we introduce some notation. Let $e\left(\sigma_{t}^{X}\right)$ be the error in the approximate sum of weights of $S_{X}$ for game $t$. Let $e\left(P L_{i}^{t}\left(S_{X}\right)\right)$ and $e\left(P W^{t}\left(S_{X}\right)\right)$ denote the errors in $P L_{i}^{t}\left(S_{X}\right)$ and $P W^{t}\left(S_{X}\right)$ respectively. These two errors are obtained in the same way as we obtained $e\left(E \Delta_{i}^{X}\right)$ in Equation 21. Hence we have:

$$
\begin{array}{r}
e\left(P L_{i}^{t}\left(S_{X}\right)\right)=\frac{1}{\sqrt{\left(2 \pi \nu^{t} / X\right)} \times \operatorname{MAX}}\left(\int_{q^{t}-\epsilon}^{q^{t}-\epsilon+e\left(\sigma_{t}^{X}\right)} e^{-X \frac{\left(x-X \mu^{t}\right)^{2}}{2 \nu^{t}}} d x,\right. \\
\left.\int_{q^{t}-w_{i}^{t}-e\left(\sigma_{t}^{X}\right)}^{q^{t}-w_{i}^{t}} e^{-X \frac{\left(x-X \mu^{t}\right)^{2}}{2 \nu^{t}}} d x\right)
\end{array}
$$

and

$$
\begin{aligned}
e\left(P W^{t}\left(S_{X}\right)\right)= & \frac{1}{\sqrt{\left(2 \pi \nu^{t} / X\right)}} \times\left(\int_{q^{t}-w^{t}-1}^{q^{t}-w_{i}^{t}-1-e\left(\sigma_{t}^{X}\right)} e^{-X \frac{\left(x-X \mu^{t}\right)^{2}}{2 \nu^{t}}} d x+\right. \\
& \left.\int_{q^{t}-e\left(\sigma_{t}^{X}\right)}^{q^{t}} e^{-X \frac{\left(x-X \mu^{t}\right)^{2}}{2 \nu^{t}}} d x\right) .
\end{aligned}
$$

For $k$-majority games, let $e\left(k E \Delta_{i}^{X}\right)$ denote the error in $i$ 's marginal contribution to a random coalition of size $X$, and let $e\left(\bar{\varphi}_{i}^{k}\right)$ denote the error in $i$ 's Shapley value. From rule $\mathrm{R} 3$, we get:

$$
e\left(k E \Delta_{i}^{X}\right)=\sum_{j=1}^{k}\left(\sum_{f=1}^{j} e\left(P L_{i}^{f}\left(S_{X}\right)\right)+\sum_{g=j+1}^{k} e\left(P W^{g}\left(S_{X}\right)\right)\right)
$$

So the error in $i$ 's Shapley value is:

$$
e\left(\bar{\varphi}_{i}^{k}\right)=\frac{1}{n} \sum_{X=1}^{n} e\left(k E \Delta_{i}^{X}\right)
$$

We now analyze the error in Banzhaf index. Combining Equation 29 with Equation 16, we get the error in $k \bar{\eta}_{i}^{X}$ as:

$$
e\left(k \bar{\eta}_{i}^{X}\right)=e\left(k E \Delta_{i}^{X}\right) \times C(n, X)
$$

The error in $k \bar{\eta}_{i}$ (see Equation 17) is:

$$
e\left(k \bar{\eta}_{i}\right)=\sum_{X=1}^{n} e\left(k \bar{\eta}_{i}^{X}\right)
$$

The error in $\bar{\beta}_{i}^{k}$ (see Equation 18) is:

$$
e\left(\bar{\beta}_{i}^{k}\right)=e\left(k \bar{\eta}_{i}\right) / 2^{n-1}
$$


The error in $\bar{\lambda}_{i}^{k}$ (see Equation 19) is:

$$
e\left(\bar{\lambda}_{i}^{k}\right)=e\left(k \bar{\eta}_{i}\right)+\sum_{i=1}^{n} e\left(k \bar{\eta}_{i}\right)
$$

From the above equations, we make the following observation regarding our methods. Observation. For a given $k$-majority game, we have the following relationship: the approximation error in a player's normalized Banzhaf index is higher than the error in its absolute Banzhaf index and the error in its Shapley value $\left(e\left(\bar{\lambda}_{i}^{k}\right)>e\left(\bar{\varphi}_{i}^{k}\right)\right.$ and $\left.e\left(\bar{\lambda}_{i}^{k}\right)>e\left(\bar{\beta}_{i}^{k}\right)\right)$.

\section{Related work}

A number of approximation methods have been proposed for finding an approximate Shapley value. These include $[9,11,19]$. The method proposed in [9] is based on computing an approximate Shapley value by making measurements on random samples of coalitions. However the method does not specify how the samples need to be drawn. It is important to know how to draw samples because this is a key factor that determines the quality of approximation. In contrast, our method is based on the approximation rule defined in Section 3.1 and does not require making measurements on random samples. The method proposed in [11] uses a different randomization method from ours but like our method, it too does not require drawing random samples. Finally, [19] presented a randomization method for an approximate Shapley value in the context of task oriented domains. Also, like our method, [11,19] have linear time complexity. Hence, in future, we need to compare the approximation error for these two methods with that for ours.

\section{Conclusions and future work}

The Shapley value and Banzhaf index are two well known indices for measuring a the power a player has in a voting game. However, the problem of computing these indices is computationally hard. To overcome this problem, we presented new approximation methods for computing these indices. Although the proposed methods have polynomial time complexity, finding an approximate Shapley value using them is easier than finding an approximate Banzhaf index. We also found the absolute error for our methods and showed that this error for the Shapley value is lower than that for the Banzhaf index. In future, we need to find the bounds on these errors.

\section{References}

1. G. Ausiello, P. Crescenzi, G. Gambosi, V. Kann, A. Marchetti-Spaccamela, and M. Protasi. Complexity and approximation: Combinatorial optimization problems and their approximability properties. Springer, 2003.

2. J. F. Banzhaf. Weighted voting doesn't work: A mathematical analysis. Rutgers Law Review, 19:317-343, 1965. 
3. P.V. Bork, H. Grote, D. Notz, and M. Regler. Data Analysis Techniques in High Energy Physics Experiments. Cambridge University Press, 1993.

4. V. Conitzer and T. Sandholm. Complexity of constructing solutions in the core based on synergies among coalitions. Artificial Intelligence Journal, 170:607-619, 2006.

5. X. Deng and C. H. Papadimitriou. On the complexity of cooperative solution concepts. Mathematics of Operations Research, 19(2):257-266, 1994.

6. E. Elkind, L. A. Goldberg, P. Goldberg, and M. Wooldridge. Computational complexity of weighted threshold games. In In Proceedings of the National Conference on Artificial Intelligence (AAAI-2007), 2007.

7. S. S. Fatima, M. Wooldridge, and N. R. Jennings. A randomized method for the Shapley value for the voting game. In Proceedings of the Sixth International Conference on Autonomous Agents and Multi-Agent Systems, pages 955-962, 2007.

8. A. Francis. Advanced Level Statistics. Stanley Thornes Publishers, 1979.

9. I. Mann and L. S. Shapley. Values for large games iv: Evaluating the electoral college by monte carlo techniques. Technical report, The RAND Corporation, Santa Monica, 1960.

10. M. J. Osborne and A. Rubinstein. A Course in Game Theory. The MIT Press, 1994.

11. G. Owen. Multilinear extensions of games. Management Science, 18(5):64-79, 1972.

12. K. Prasad and J. S. Kelly. NP-completeness of some problems concerning voting games. International Journal of Game Theory, 19:1-9, 1990.

13. T. Rahwan and N. R. Jennings. An algorithm for distributing coalitional value calculations among cooperating agents. Artificial Intelligence Journal, 171:535-567, 2007.

14. T. Sandholm and V. Lesser. Coalitions among computationally bounded agents. Artificial Intelligence Journal, 94(1):99-137, 1997.

15. L. S. Shapley. A value for $\mathrm{n}$ person games. In A. E. Roth, editor, The Shapley value, pages 31-40. University of Cambridge Press, Cambridge, 1988.

16. O. Shehory and S. Kraus. A kernel-oriented model for coalition-formation in general environments: Implemetation and results. In In Proceedings of the National Conference on Artificial Intelligence (AAAI-96), pages 131-140, 1996.

17. O. Shehory and S. Kraus. Methods for task allocation via agent coalition formation. Artificial Intelligence Journal, 101(2):165-200, 1998.

18. J. R. Taylor. An introduction to error analysis: The study of uncertainties in physical measurements. University Science Books, 1982.

19. G. Zlotkin and J. Rosenschein. Coalition, cryptography, and stability: mechanisms foe coalition formation in task oriented domains. In In Proceedings of the National Conference on Artificial Intelligence (AAAI-94), pages 432-437, 1994. 